\title{
Application of Numerical Noise Titration during Autonomic Blockade
}

\author{
S Vandeput ${ }^{1}$, F Beckers ${ }^{2}$, B Verheyden ${ }^{2}$, AE Aubert ${ }^{2}$, S Van Huffel ${ }^{1}$ \\ ${ }^{1}$ Department of Electrical Engineering, ESAT-SCD, Katholieke Universiteit Leuven, Belgium \\ ${ }^{2}$ Laboratory of Experimental Cardiology, Faculty of Medicine, Katholieke Universiteit Leuven, \\ Belgium
}

\begin{abstract}
The influence of pharmacological autonomic nervous system interventions on nonlinear cardiovascular regulatory indices is nowadays still not clear. The present study assesses the effects of autonomic blockade ( $\alpha$ - and $\beta$-adrenoceptor and cholinergic) on cardiovascular function by heart rate variability (HRV) and blood pressure variability $(B P V)$ in rats using the nonlinear numerical noise titration technique. The results show that the $\alpha$ adrenoceptor pathways seem to have an important contribution in the generation and control of nonlinear $H R$ and especially BP fluctuations, while $\beta$-adrenoceptor and cholinergic interventions have a negligible contribution to the nonlinear dynamical control.
\end{abstract}

\section{Introduction}

Since the initial publication by Akselrod et al. in 1981 [1] describing the relationship between spectral components of heart rate variability (HRV) and sympathetic and vagal modulation, many studies have emerged describing this influence of the autonomic nervous system (ANS) on HRV $[2,3]$. But not only the power spectral density description of HRV can be used to detect regularity or irregularity of cardiovascular function, analysis of nonlinear dynamics results in important information too.

The nonlinear control of heart rate (HR) and blood pressure (BP) makes it physiologically easier to adapt faster and more subtly to changes in physiological needs. Analysis methods derived from nonlinear system dynamics have opened a new approach for studying and understanding the characteristics of cardiovascular dynamics. Conventional spectral analysis of HRV and BPV can provide analytical features of its cyclic variation but cannot show the dynamical properties of the fluctuations. Nonlinear methods are typically designed to assess the quality, scaling and correlation properties, rather than to assess the magnitude of variability like conventional HRV methods do. Furthermore, it has been shown that ANS control underlies the nonlinearity and possible chaos of normal HRV [4].
Due to the increased use of nonlinear dynamics in clinical studies, it is very important to understand the physiological mechanisms underlying the generation of these fluctuations. Changes in nonlinear behaviour can already be found in several pathologies, but the exact contributions of the nonlinear indices in these cases need to be investigated further.

We hypothesize here that nonlinear cardiovascular fluctuations are generated by the autonomic nervous system. Suppressing the activity of one branch of the autonomic nervous system using pharmacological blockades should alter the nonlinear characteristics of HR and BP fluctuations in order to compensate for the disturbance of the equilibrated system. Using different types of autonomic blocking agents in rats allows the examination of the involvement of the sympathetic and vagal nervous system in the generation of nonlinear cardiovascular fluctuations.

During the last decade, studies using robust nonlinear detection techniques have provided some of the strongest support for the presence of chaos in HRV [5]. Specifically, the method of noise titration $[6,7]$ provides a highly sensitive test for deterministic chaos and a relative measure for tracking chaos of a noise-contaminated signal in short data segments.

In the present study, the influence of different pharmacological interventions was investigated in rats on chaotic dynamics by applying the numerical noise titration algorithm to RR interval (RRI) and BP time series. The aim is to examine the influence of several autonomic blocking agents on nonlinear HRV and BPV indices, and more specifically to detect and quantify changes in deterministic chaos of HRV and BPV in response to changes in autonomic control.

\section{Methods}

\subsection{Data acquisition}

13 male Wistar rats were conditioned in a Plexiglas triangular restrainer for at least 2 weeks before the actual experiment. On the day of the experiment, 3 precordial elec- 
trocardiogram electrodes were inserted subcutaneously under short halothane anaesthesia and with artificial ventilation. After termination of anaesthesia, rats were placed in the plexiglas restrainer in a quiet room with dimmed lights and were allowed to recover for several hours (minimum 2 hours). This was sufficiently long to exclude the effect of anaesthesia. After checking blood gases to exclude respiratory acidosis and obtaining values within the normal range, the recordings were performed.

Blockade of $\alpha$-and $\beta$-adrenoceptors was achieved using the intravenous administration, over $1 \mathrm{~min}$, of phentolamine or propanolol respectively, while atropine sulphate was used for the cholinergic blockade. Recordings were made up to $30 \mathrm{~min}$ after the intravenous administration of the antagonist(s). Of the group of 13 rats, 4 received $\alpha$-adrenoceptor blockade, 5 received $\beta$-adrenoceptor blockade and 4 received cholinergic blockade without crossover.

Recordings of at least 20 min after autonomic blockade were sufficiently long to calculate nonlinear indices because of the high HR in rats. Each recording contained at least $7000 \mathrm{RR}$ intervals. The sampling rate was 1000 $\mathrm{Hz}$ per channel. The RR intervals and systolic BP values were calculated [8] and, after visual inspection, exported for further analysis.

More details concerning the way the rats were conditioned and the signals were measured, are described in [9].

\subsection{Algorithm}

The different parts of the numerical noise titration algorithm are well described in [10].

\section{Modeling.}

For any heartbeat RR time series $y_{n}, n=1,2, \cdots, N$, a closed-loop version of the dynamics is proposed in which the output $y_{n}$ feeds back as a delayed input. The univariate time series are analysed by using a discrete Volterra autoregressive series of degree $d$ and memory $\kappa$ as a model to calculate the predicted time series $y_{n}^{\text {calc. }}$ :

$$
\begin{aligned}
y_{n}^{\text {calc }} & =a_{0}+a_{1} y_{n-1}+a_{2} y_{n-2}+\cdots+a_{\kappa} y_{n-\kappa} \\
& +a_{\kappa+1} y_{n-1}^{2}+a_{\kappa+2} y_{n-1} y_{n-2}+\cdots \\
& +a_{M} y_{n-\kappa}^{d} \\
& =\sum_{m=1}^{M-1} a_{m} z_{m}(n)
\end{aligned}
$$

where $\mathbf{M}=(\kappa+d) ! /(\kappa ! d !)$ is the total dimension. Thus, each model is parameterised by $\kappa$ and $d$ which correspond to the embedding dimension and the degree of the nonlinearity of the model (i.e. $d=1$ for linear and $d>1$ for nonlinear model). The coefficients $a_{m}$ are recursively estimated from (1) by using the Korenberg algorithm.

\section{Nonlinear detection (NLD).}

The goodness of fit of a model (linear vs. nonlinear) is measured by the normalised residual sum of squared errors:

$$
\varepsilon(\kappa, d)^{2}=\frac{\sum_{n=1}^{N}\left(y_{n}^{\text {calc }}(\kappa, d)-y_{n}\right)^{2}}{\sum_{n=1}^{N}\left(y_{n}-\mu_{y}\right)^{2}}
$$

where $\mu_{y}=1 / N \sum_{n=1}^{N} y_{n}$ and $\varepsilon(\kappa, d)^{2}$ represents a normalised variance of the error residuals. The optimal model $\left\{\kappa_{o p t}, d_{o p t}\right\}$ is the model that minimizes the Akaike information criterion:

$$
C(r)=\log \varepsilon(r)+r / N
$$

where $r \in[1, M]$ is the number of polynomial terms of the truncated Volterra expansion from a certain pair $(\kappa, d)$.

\section{Numerical noise titration.}

The NLD is used to measure the chaotic dynamics inherent in the RR series by means of numerical noise titration as follows:

1. Given a time series $y_{n}$, apply the NLD to detect nonlinear determinism. If linear, then there is insufficient evidence for chaos.

2. If nonlinear, it may be chaotic or non-chaotic. To discriminate these possibilities, add a small $(<1 \%$ of signal power) amount of random white noise to the data and then apply NLD again to the noise corrupted data. If linear, the noise limit (NL) of the data is zero and the signal is nonchaotic.

3. If nonlinearity is detected, increase the level of added noise and again apply NLD.

4. Repeat the above step until nonlinearity can no longer be detected when the noise is too high (low signal-to-noise ratio). The maximum noise level (i.e. NL) that can be added to the data before nonlinearity can not be detected, is directly related to the Lyapunov exponent (LE).

\section{Decision tool.}

Under this numerical titration scheme, NL $>0$ indicates the presence of chaos, and the value of NL gives an estimate of relative chaotic intensity. Conversely, if $\mathrm{NL}=$ 0 , then the time series may be non-chaotic or the chaotic component is already neutralised by the background noise. Therefore, the condition NL $>0$ provides a simple sufficient test for chaos. Details of NLD and numerical noise titration are discussed in $[6,7]$.

\subsection{Analysis}

Numerical noise titration was applied on the resampled $(10 \mathrm{~Hz}) \mathrm{RRI}$ and BP time series using a 60-second window and sliding the window every 5 seconds. Statistical 


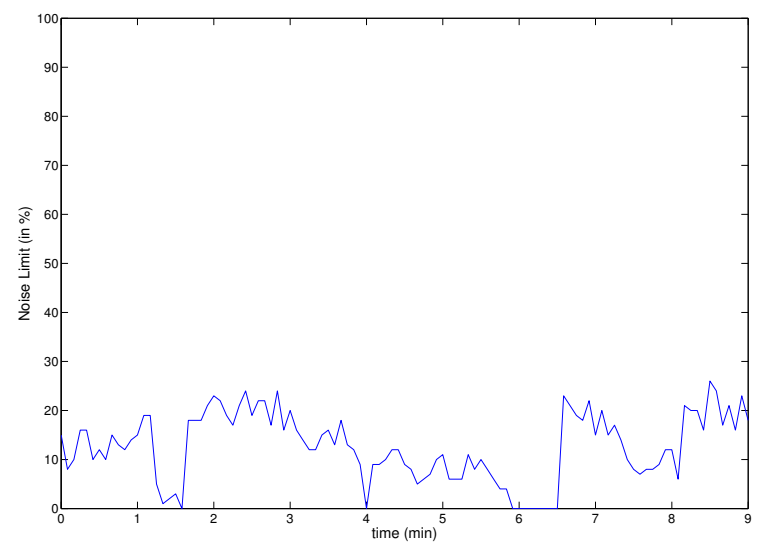

Figure 1. Typical example of NL values after applying the noise titration technique to RRI time series with cholinergic blockade.

comparison between means within groups, before and after intravenous pharmacological blockade, was analysed by paired Student's t-tests. Differences between groups were analysed in general by the Kruskal-Wallis test and more in detail by unpaired Student's t-tests between 2 groups. $\mathrm{P}<$ 0.05 was considered statistically significant.

\section{Results}

A typical result of applying the noise titration technique to a time series is shown in figure 1. It illustrates also that the Noise Limit (NL) values can fluctuate strongly in time, even for the same subject under the same physiological conditions.

Table 1. P-values of pairwise comparison of mean NL between normal condition and pharmacological blockade for HR and BP.

\begin{tabular}{lcr}
\hline \hline groups & HR & BP \\
\hline$\alpha$-blockade vs. normal & 0.3209 & $\mathbf{0 . 0 0 5 1}$ \\
$\beta$-blockade vs. normal & 0.2775 & 0.5714 \\
cholinergic blockade vs. normal & 0.3882 & 0.4774 \\
\hline \hline
\end{tabular}

For this reason, we calculated the mean NL value for each rat under each condition. Within each group, this mean NL under a specific pharmacological blockade was compared pairwise with that of the corresponding normal control group. The results for the different groups are plotted by means of boxplots in figure 2 and the p-values of the statistical analysis are shown in table 1 .

Next, we compared the same measure between the different groups. Figure 3 indicates that an $\alpha$-adrenoceptor
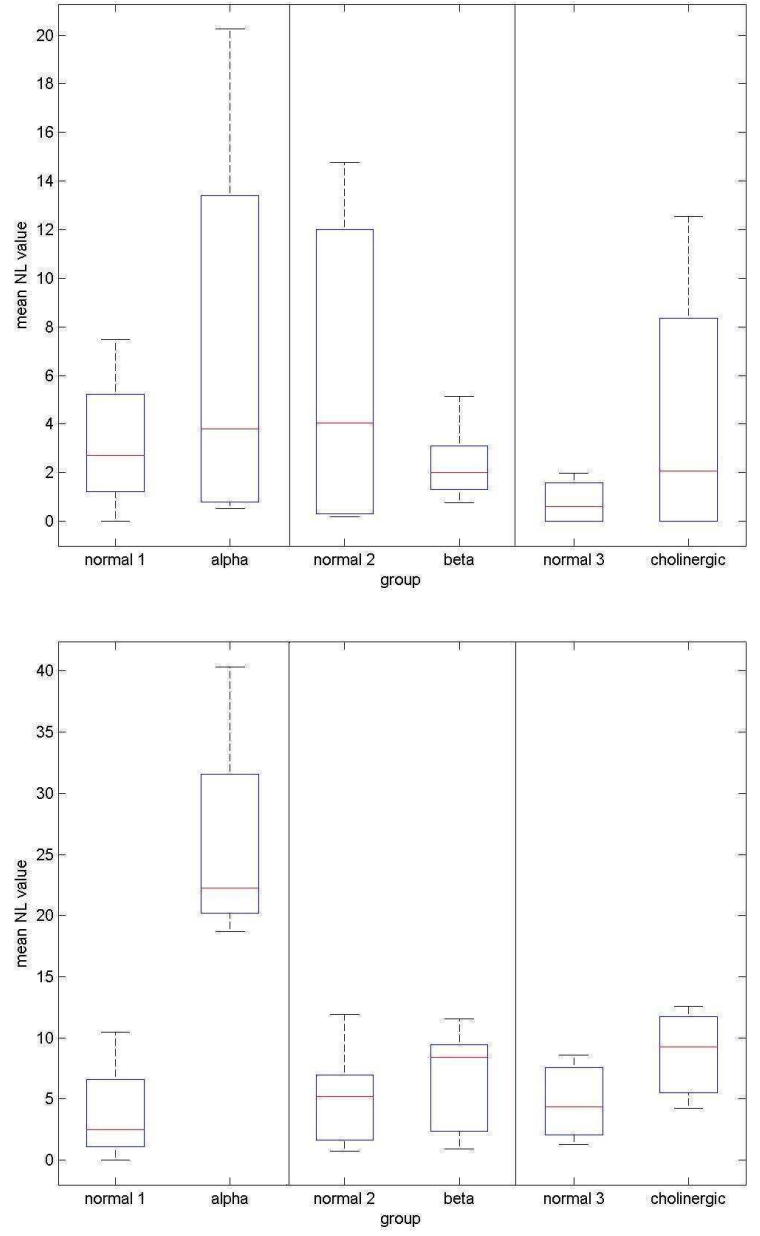

Figure 2. Boxplots to compare pairwise different groups of pharmacological blockade with their corresponding normal control group for HRV (top) and BPV (bottom)

blockade leads to a higher mean NL, in particular in case of BP. The Kruskal-Wallis test gives $\mathrm{p}=0.8625$ for HRV and $\mathrm{p}=0.0096$ for BPV. Table 2 shows for the BP signal the original p-values between 2 groups, confirming the discriminating character to distinguish the $\alpha$-adrenoceptor blockade group from any other group. Even after correction for multiple testing, statistical significance remains.

\section{Discussion and conclusions}

The present results support, partially, the hypothesis that disturbing the autonomic nervous system induces changes in the nonlinear modulation of HR and BP, but in a complicated way. In rats, the $\alpha$-adrenoceptor pathways seem to have an important contribution in the generation and control of nonlinear HR and especially BP fluctuations, while $\beta$-adrenoceptor and cholinergic interventions have a negli- 

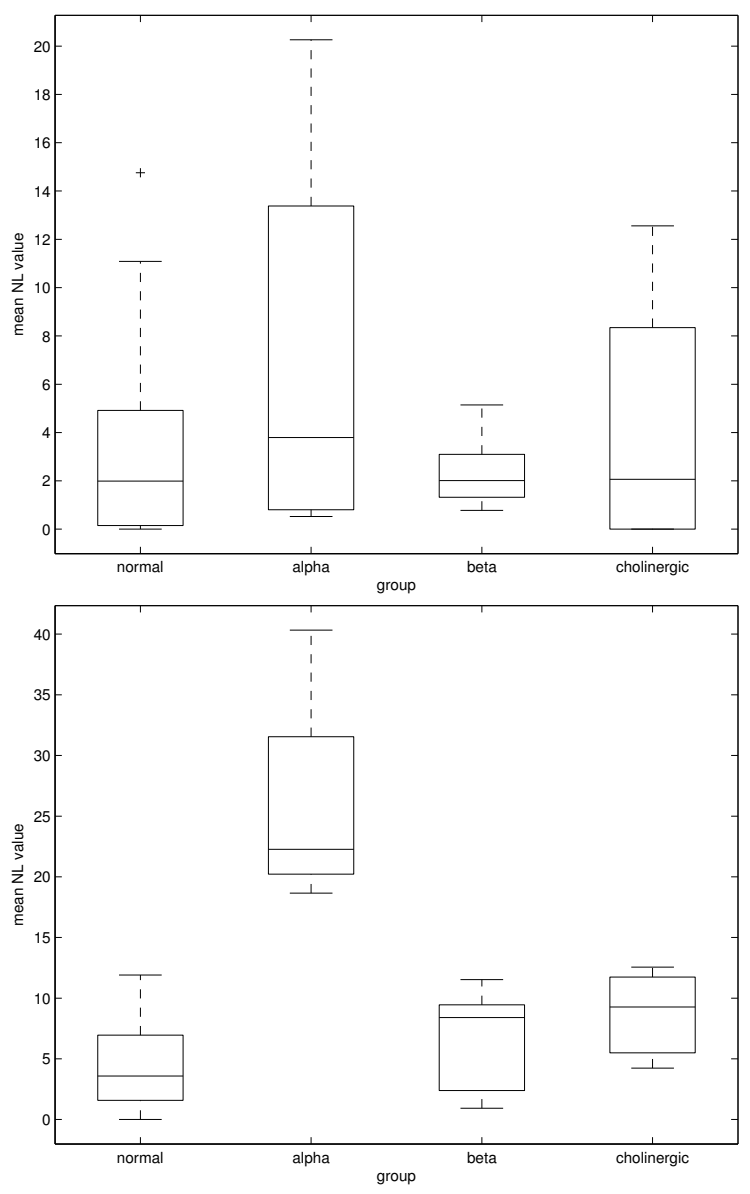

Figure 3. Boxplots to compare between different groups for HRV (top) and BPV (bottom).

Table 2. Original p-values of comparison between different groups for BPV.

\begin{tabular}{l|cccc}
$\mathrm{p}$ & normal & $\alpha$ & $\beta$ & cholinergic \\
\hline normal & 1 & $\mathbf{0 . 0 1 9}$ & 0.387 & 0.210 \\
$\alpha$ & & 1 & $\mathbf{0 . 0 2 2}$ & $\mathbf{0 . 0 3 2}$ \\
$\beta$ & & & 1 & 0.517 \\
cholinergic & & & & 1
\end{tabular}

gible contribution to the nonlinear dynamical control.

Not only the mean NL was used as measure in this study, but also the median NL and several proportionality measures. All reflected the same conclusions.

A critical remark can be made concerning some limitations of the study. Only a limited number of rats could be used for the present study for ethical reasons. Moreover, a pharmacological blockade drives the cardiovascular system out of the physiological range. In addition, a nonlinear method can not be easily visualised and needs to be interpreted in a theoretical way.
An important contribution of this work is our finding that the numerical noise titration technique seems very sensitive to changes in vasomotion control. This might help to understand the physiological origin of nonlinear blood pressure control.

\section{Acknowledgements}

Research supported by GOAAMBioRICS, CoE EF/05/006, IUAP P6/04, BIOPATTERN (FP6-2002-IST 508803), ETUMOUR (FP6-2002-LIFESCIHEALTH 503094), Healthagents (IST-2004-27214), FAST (FP6-MC-RTN-035801) and ESA (Prodex-8 C90242).

Steven Vandeput and Bart Verheyden are supported by the Belgian Federal Office of Scientific Affairs (ESAPRODEX). Frank Beckers is a postdoctoral researcher of the Research Fund K.U.Leuven.

\section{References}

[1] Akselrod S, Gordon D, Ubel FA, Shannon DC, Berger AC, Cohen RJ. Power spectrum analysis of heart rate fluctuation: A quantitative probe of beat-to-beat cardiovascular control. Science 1981;213:220-222.

[2] Malliani A, Pagani M, Lombardi F, Cerutti S. Cardiovascular neural regulation explored in the frequency domain. Circulation 1991;84:482-492.

[3] Eckberg DL. Sympathovagal balance: A critical appraisal. Circulation 1997;96:3224-3232.

[4] Goldberger AL, Rigney DR, West BJ. Chaos and fractals in human physiology. Sci Am 1990;262:43-49.

[5] Poon CS, Merrill CK. Decrease of cardiac chaos in congestive heart failure. Nature 1997;389:492-495.

[6] Poon CS, Barahona M. Titration of chaos with added noise. PNAS USA 2001;98:7107-7112.

[7] Barahona M, Poon CS. Detection of nonlinear dynamics in short, noisy data. Nature 1996;381:215-217.

[8] Beckers F, Ramaekers D, Aubert AE. Acts: Automated calculation of tachograms and systograms. Prog Biomed Res 1999;4:160-165.

[9] Beckers F, Verheyden B, Ramaekers D, Swynghedauw B, Aubert AE. Effects of autonomic blockade on non-linear cardiovascular variability indices in rats. Clinical and Experimental Pharmacology and Physiology 2006;33:431439.

[10] Zapanta L, Poon CS, White DP, Marcus CL, Katz ES. Heart rate chaos in obstructive sleep apnea in children. In Proceedings of the 26th Annual IEEE Engineering in Medicine and Biology Conference. San Francisco, USA: IEEE EMBS, 2004; 3889-3892.

Address for correspondence:

Steven Vandeput

ESAT-SCD, Kasteelpark Arenberg 10, 3001 Leuven, Belgium steven.vandeput@esat.kuleuven.be 